\title{
Characterising Meanders Qualitatively
}

\author{
Björn Gottfried \\ Artificial Intelligence Group, TZI \\ University of Bremen, Germany \\ bg@tzi.de
}

\begin{abstract}
In this paper, a qualitative shape representation is described for the purpose of characterising linear geographical and artificial objects. In particular, we focus on the curve progression telling us how objects spread across the landscape. For instance, sinuosities of rivers provide important information about imperilled locations in the case of flood waters. However, precise geometrical descriptions are overdetermined and frequently difficult or sometimes impossible to obtain. By contrast, we introduce a concept which allows curves to be classified on the basis of a qualitative representation that defines properties of linear objects, which derive from how segments of objects are located relative to other segments, arriving at conclusions such as how twisty a curve is. Especially, the new method can be applied if only coarse information is available and even then if objects are given incompletely.
\end{abstract}

\section{Introduction}

In geographical information systems topological relations between geographical objects are useful [3. It may, for example, be crucial to a particular query that there is a forest and that there is a river which is not connected to the forest; but it does not matter at all what the boundary of the forest looks like, or how far the river is from the forest provided that they are not in contact; such geometrical relationships are not important when we are interested in those cases where only the given topological relationships hold. Precise correspondences would retrieve fewer results than there are actually in the database. But sometimes topological relationships do not sufficiently characterise the query. For example, it might be crucial to take the curve progression of the river into account. How can different meanders of rivers, such as those in Fig. 1 be described? Similarly, there are many kinds of geographical and artificial objects for which curvature information is important, including among others contour lines in topographic maps, coastlines, borders of countries and other regions, transportation networks, such as roads and railways, irrigation networks, and sewer systems.

Modern geographic information systems demand concepts that provide means which are closely related to how people deal with spatial information, since it is desirable that user interfaces become more natural. Capturing commonsense knowledge about objects, qualitative representations lend themselves to provide 


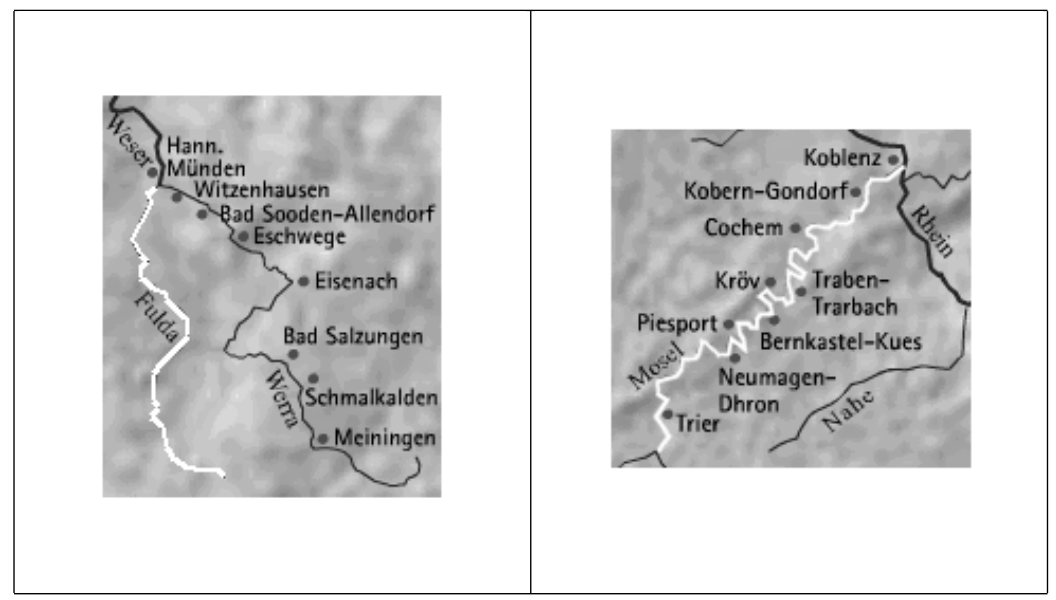

Fig. 1. Rivers differing in their curvatures

appropriate representational concepts 2]. Especially, qualitative boundary based approaches which are related to linear objects record features of boundaries while walking along them. Those which have been devised most recently include [6 11 14 10] [11] base their approach on a discretisation of tangent bearing and curvature, considering the rate of change of curvature, and they give a set of tokens from which higher-level tokens can be derived. By contrast, all other approaches rely on polygonal approximations of the underlying boundaries. There are several reasons for this. Most importantly, polygons are at the core of geographical information systems; the discrete space used in computer representations inherently deals with polygons; depending on the application at hand objects can be approximated at a number of different granularity levels when using polygons; frequently, polygons are directly given (when recording the path of a navigating system the position of which is measured at intervals, for example); polygons provide concise representations of even highly complex objects, forming an appropriate basis for the qualitative characterisation of shapes from both the local and global points of view. Considering linear objects such as rivers, we are actually concerned with open polygons, i.e. simple polylines. Below, we simply speak of polygons, denoting both simple polylines and simple polygons.

This paper addresses the problem of how to represent smooth curves by polygons in such a way that the curve progression of linear objects is made explicit. Improving user interfaces in such a manner that distinctions made by the system are easily comprehensible by humans, a qualitative representation is recommended. As such section 2 introduces the qualitative representation on which we shall define qualitative features for the purpose of characterising meanders of linear objects in section 3 . A number of examples in section 4 illustrate 


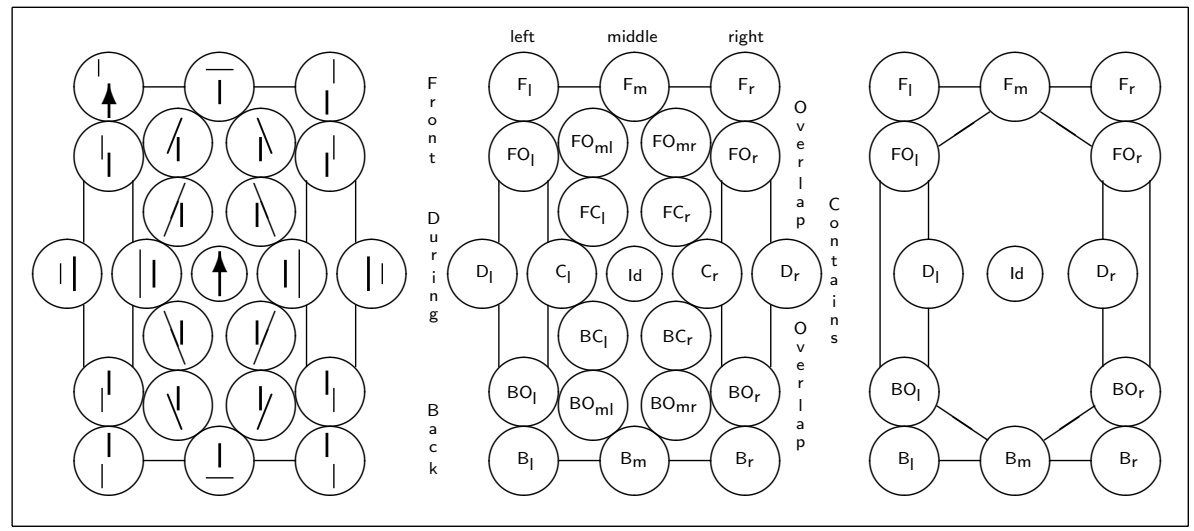

Fig. 2. The conceptual neighbourhood graphs of $\mathcal{B} \mathcal{A}_{23}$ (middle) and $\mathcal{B} \mathcal{A}_{13}$ (right)

how those qualitative features apply to linear objects. An extended concept of neighbourhood relations is introduced in section 5 , which forms the basis for the introduced concepts and which generalises to other qualitative representations. We conclude in section 6 , suggest topics for future research, and summarise in section 7 .

\section{A Qualitative Feature Scheme}

In this section we will summarise previous work on a qualitative feature scheme on which the current approach is based. This feature scheme consists of a number of relations which describe arrangements between line segments in two dimensions. Such relations have been referred to as bipartite arrangements, $\mathcal{B A}$ for short [7. Fig. 2 shows these relations.

If one line of a polygon is made the basis, the position of every other line can be described relative to it, using the relations of $\mathcal{B A}_{23}$ which are shown on the left hand side of Fig. 2. In this way, the qualitative context of a polygonal line, $\mathrm{x}$, is considered, and for a polygon with $\mathrm{n}$ lines we obtain a list of relations to which we refer to as the course of reference segment $\mathrm{x}$, in short $\mathrm{C}(\mathrm{x})$ :

$$
\mathrm{C}(\mathrm{x}) \equiv\left(\mathrm{x}_{\mathrm{y}_{1}}, . ., \mathrm{x}_{\mathrm{y}_{\mathrm{n}}}\right), \mathrm{x}_{\mathrm{y}_{\mathrm{i}}} \in \mathcal{B \mathcal { A }}_{23}, \mathrm{i}=1, . ., \mathrm{n}
$$

with $x_{y_{1}}$ meaning that line segment $y_{1}$ is described with respect to reference segment $\mathrm{x} 11$ In particular, it holds that $\mathrm{x}_{\mathrm{x}}=\mathrm{Id}$. A subset $\mathcal{B A}_{13} \subset \mathcal{B A}_{23}$, shown on the right hand side of Fig. 2. provides a set of atomic relations in the sense that all other relations can be obtained by combining $\mathcal{B A}_{13}$ relations (e.g. $\left.\mathrm{C}_{\mathrm{I}}=\mathrm{BO}_{1} \mathrm{D}_{1} \mathrm{FO}_{1}\right)$.

\footnotetext{
${ }^{1}$ Instead of the common infix notation we use indices in order to be able to list many $\mathcal{B A}_{23}$ relations in a compact way.
} 
The entire range of relations (accordingly to $\mathcal{B A}_{13}$ ) where $\mathrm{C}(\mathrm{x})$ runs along is called its scope, and the number of different $\mathcal{B A}_{13}$ relations involved is called the extent, $\eta(\mathrm{C}(\mathrm{x}))$ for short. The shortest extent is 0 in which case there is no other line segment than the reference segment $x$ itself; the largest possible extent is 12 (which is $\left|\mathcal{B A}_{13} \backslash\{\operatorname{ld}\}\right|$ ) in which case the course runs completely around $\mathrm{x}$. Scopes of such courses are also referred to as universal scopes since all relations of $\mathcal{B A}_{23}$ are realisable within these scopes. Eventually, there exist different scopes which have the same extent, for instance, one course may go from $F_{\mid}$to $F_{r}$ and another one from $B_{\mid}$to $B_{r}$; both courses, however, have an extent of 3.

While 9] distinguishes local and global features, here we simplify matters by representing both local and global features by bipartite arrangements. That is, we conceive each pair of line segments in a simple polygon as to be free of intersections, meaning that especially adjacent line segments have no point in common. For this purpose, we stipulate that each point which connects two line segments belongs to the first line segment in an oriented polygon. This enables us to get by with $\mathcal{B A}_{23}$ relations, as depicted in Fig. 2,

Having a polygon with $\mathrm{n}$ line segments there exist $\mathrm{n}$ courses, each one comprising $\mathrm{n}$ bipartite relations, i.e. such a feature scheme comprises a total of $\mathrm{n}^{2}$ relations. Writing down all courses, one below the other, for a polygon with six lines the following matrix is obtained (compare the polygon on the left hand side of Fig. 3, and note that singular relations, such as between $\mathrm{x}$ and $\mathrm{y}$, are dealt with in accordance to $[8]$ ):

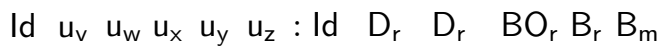

$$
\begin{aligned}
& \begin{array}{lllllllllllll}
v_{u} & \text { Id } & v_{w} & v_{x} & v_{y} & v_{z}: D_{r} & \text { Id } & F_{l} & F_{m} & F_{r} & C_{r}
\end{array} \\
& w_{u} w_{v} \text { Id } w_{x} w_{y} w_{z}: B_{m} B_{l} \text { Id } F_{r} C_{r} B_{r} \\
& \begin{array}{llllllllllllllll}
x_{u} & x_{v} & x_{w} & l d & x_{y} & x_{z} & : & B_{r} & B_{r} & B_{r} & \text { ld } & D_{r} & D_{r}
\end{array}
\end{aligned}
$$

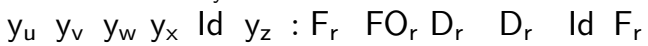

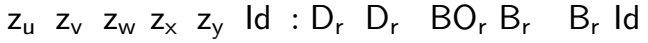

\section{Towards the Characterisation of Meanders}

A bend in a river is referred to as a meander, emerging from a stream flowing through a wide valley or flat plain thereby tending to form a meandering stream course as it alternatively erodes and deposites sediments along its course. The result is a snaking pattern as the stream meanders back and forth across its floodplain.

In the following, we shall generalise this concept, using the term meander broadly for linear objects which are shaped by several twists and turns, and we will characterise winding courses, i.e. how a polygon develops from the point of view of one of its line segments, to which we will refer to as the reference segment. The simplest way of characterising a course consists in referring to its $\mathcal{B A}_{23}$ relations. But what does a list of many $\mathcal{B A}_{23}$ relations tell us? (Have a look at the matrix above.) We shall rather identify more abstract features 


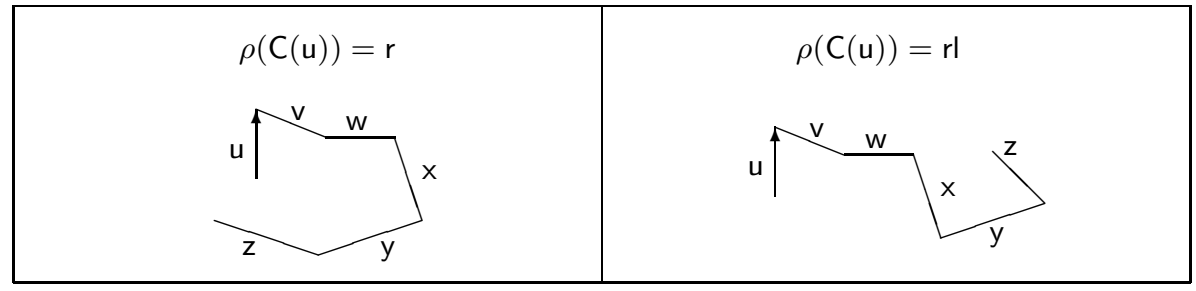

Fig. 3. Circulation directions around a reference line

which derive from this matrix, and which concisely describe a course, such as whether it runs around the reference segment clockwise or anticlockwise, whether it comprises reversals, and towards which directions the course runs along. In this way we will characterise differently shaped meanders.

Formally, at the finest level sequences of $\mathcal{B A}_{23}$ relations are considered (equation (1), and at a coarser level sequences of generalised directions are considered (section 3.1). In the latter case atomic $\mathcal{B A}_{23}$ relations are put together in order to form yet a coarser description than $\mathcal{B A}_{23}$ relations. Following a list of $\mathcal{B A}_{23}$ relations or generalised directions a course either orbits clockwise or anticlockwise around the reference segment (section 3.2). Taking the first derivative of a course amounts to consider changes in direction to which we refer to as reversals (section 3.3). Positions at which such changes occur are analogous to local minima and maxima of functions and they are referred to as inflection segments (section 3.4).

\subsection{Generalised Directions}

Suppose a polygon is described with respect to one of its line segments, $x$. As far as we are only interested in the overall direction towards which the course of $x$ runs along, different $\mathcal{B A}_{23}$ relations can be put together which make up the same direction at a coarser granularity level. Such coarser directions form subsets of $\mathcal{B A}_{23}$. Leaving out the identity relation, there are $2^{22}=4194304$ such subsets. Some of them are particularly useful as coarse directions; for example, a course may run somewhere left of the reference segment:

$$
C(x)=I \equiv \forall_{y \neq x}: x_{y} \in\left\{F_{1}, F_{1}, D_{1}, C_{1}, B_{1}, B_{l}\right\}
$$

These six relations combine to $2^{6}=64$ different sets which form the basis of several different courses, all of them running left of the reference segment. $C(x)=r$ (right of), $\mathrm{C}(\mathrm{x})=\mathrm{F}$ (in front of), $\mathrm{C}(\mathrm{x})=\mathrm{B}$ (back of), etc., can be defined in the same way. $B y$ this means, complex concepts are based on fewer relations than when taking individual $\mathcal{B A}_{23}$ relations. Altogether, we introduce generalised directions as to be defined over sets of atomic $\mathcal{B A}_{13}$ relations: 


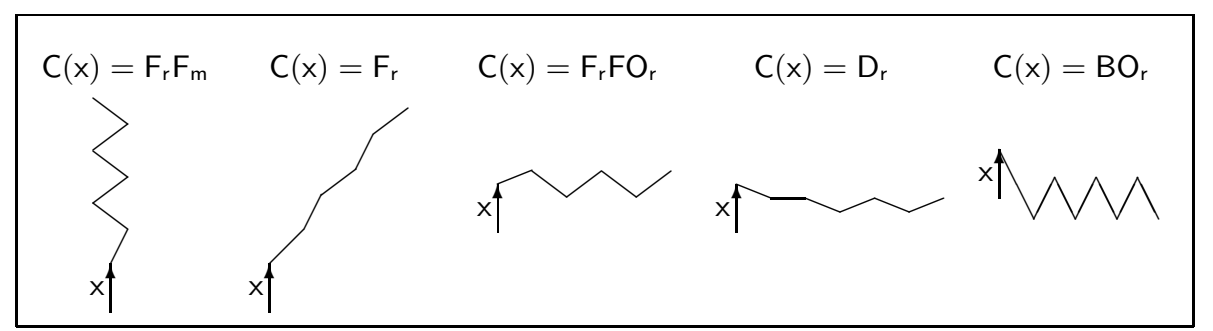

Fig. 4. Five polygons with three of them leading in a single direction $\left(F_{r}, D_{r}\right.$, and $\mathrm{BO}_{\mathrm{r}}$ ) with respect to line segment $\mathrm{x}$, while two of them are almost limited to one direction

\section{Definition 1 (Generalised direction)}

$\mathrm{x}$ is line segment of a polygon and $\mathrm{C}(\mathrm{x})$ is its course. A generalised direction of $\mathrm{C}(\mathrm{x}), \omega(\mathrm{C}(\mathrm{x}))$, is a set $\mathrm{M} \in \mathcal{P}\left(\mathcal{B A}_{13}\right)$.

For instance, the right hand side of Fig. 3 shows a polygon which can be described as to run (somehow) right of $\mathrm{x}$, thus $\omega(\mathrm{C}(\mathrm{x}))=\mathrm{r}$; but this is not entirely true for the polygon on the left hand side.

How do generalised directions of different reference segments combine? Taking into account simultaneously the directions of all courses, properties such as the convexity of closed polygons can be derived. For an arbitrary closed polygon, P, which is oriented anticlockwise it holds

\section{Proposition 1}

$\operatorname{convex}(\mathrm{P}) \Leftrightarrow \forall_{\mathrm{x} \in \mathrm{P}}: \omega(\mathrm{C}(\mathrm{x}))=\mathrm{I}$

Proof: reduction on the triangle orientation: for three adjacent points of a convex polygon $\mathrm{P}$ which is oriented anticlockwise, their triangle orientation is anticlockwise. Accordingly, the first two points define a reference segment, $x$, while the third point lies left of this line, as does each following point. As a consequence, each line segment, $y$, defined by two such following points, lies left of $x$. As x can be defined by two arbitrary points of $\mathrm{P}$, for each course it holds that all its line segments lie left of the reference segment, and it holds that $\forall_{x \in P}: \omega(C(x))=I$.

Conversely, if $\forall_{x \in P}: \omega(C(x))=1$, then for each pair of lines it holds that the primary segment lies left of the reference segment. The same holds for the endpoints of such pairs of line segments, which define triangle orientations which are all oriented anticlockwise, indicating that the polygon is convex.

While both $\mathcal{B A}_{23}$ relations and generalised directions describe how parts of a polygon are located relative to other parts of that same polygon, considering meanders we are interested in how these relative locations change when following the courses of the reference segments. 


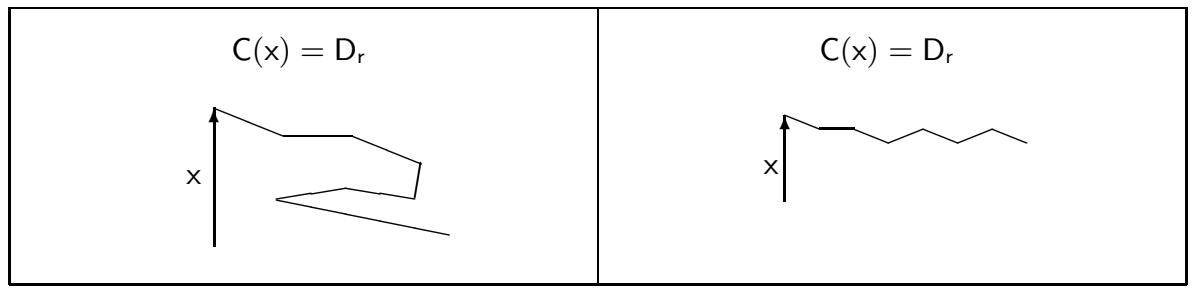

Fig. 5. Two polygons describing the same course with respect to line segment $x$

\subsection{Circulation Direction}

Irrespective of generalised directions introduced in the previous section, it can be distinguished whether a course circulates left around or right around the reference segment:

\section{Definition 2 (Circulation direction)}

$\mathrm{x}$ is line segment of a polygon. The circulation between two neighbouring line segments, $\mathrm{y}$ and $\mathrm{y}^{\prime}$, is left of $\mathrm{x}$, i.e. $\rho\left(\mathrm{x}_{\mathrm{y}}, \mathrm{x}_{\mathrm{y}^{\prime}}\right)=\mathrm{I}$, if the path from the first relation $\mathrm{x}_{\mathrm{y}}$ to the second relation $\mathrm{x}_{\mathrm{y}^{\prime}}$ runs anticlockwise around $\mathrm{x}$; otherwise, it is right of $\mathrm{x}$, in short $\mathrm{r}$. If the direction does not change it holds that $\rho\left(\mathrm{x}_{\mathrm{y}}, \mathrm{x}_{\mathrm{y}^{\prime}}\right)=\varepsilon$, as it does if $\mathrm{y}$ and $\mathrm{y}^{\prime}$ are not adjacent line segments.

$\rho\left(\mathrm{x}_{\mathrm{y}}, \mathrm{x}_{\mathrm{z}}\right)=\varepsilon$ denotes the case that it cannot be determined from the point of view of $x$ whether the circulation direction from $y$ to $z$ is clockwise or anticlockwise with respect to $x$. This indeterminacy can either be compensated by another reference segment, or $y$ and $z$ are not adjacent in which case their circulation direction cannot be derived from y and $z$ alone.

Fig. 3 shows two examples. On the left hand side the course of $x$ circulates entirely right of $\mathrm{x}$. On the right hand side it also runs right of $\mathrm{x}$, but it then turns back and as a consequence runs left of $x$. Note how this is different from the generalised direction $\omega(\mathrm{C}(\mathrm{x}))=\mathrm{r}$.

Further examples clarify the meaning of the circulation direction: $\rho\left(\mathrm{F}_{\mathrm{I}} \mathrm{FO}_{\mathrm{I}}\right)=\mathrm{I}$, $\rho\left(\mathrm{FO}_{\mathrm{l}} \mathrm{F}_{\mathrm{l}}\right)=\mathrm{r}, \quad \rho\left(\mathrm{F}_{\mathrm{l}} \mathrm{F}_{\mathrm{m}}\right)=\mathrm{r}, \quad \rho\left(\mathrm{F}_{\mathrm{l}} \mathrm{FO}_{\mathrm{mr}} \mathrm{BO}_{\mathrm{r}}\right)=\mathrm{rr}, \quad \rho\left(\mathrm{F}_{\mathrm{l}} \mathrm{FO}_{\mathrm{mr}} \mathrm{FO}_{\mathrm{r}} \mathrm{FO}_{\mathrm{r}} \mathrm{D}_{\mathrm{r}}\right)=\mathrm{rlr}$, and $\rho\left(\mathrm{FO}_{\mathbf{l}} \mathrm{F}_{\mathbf{l}} \mathrm{IdD}_{\mathrm{r}} \mathrm{BO}_{\mathrm{r}}\right)=\mathrm{rr}$. Equal neighbouring directions can be omitted in order to obtain only the changes, i.e. changes between left and right, or anticlockwise and clockwise, respectively. It then holds that the number of changes between left and right of $\rho(\mathrm{C}(\mathrm{x}))$ is less than or equal to the length of the course.

Taking each of the line segments of the polygon on the left hand side of Fig. 3 as a reference segment, the circulation direction is always either $r$ (clockwise) or $\varepsilon$ (indeterminate). But the circulation directions of different reference segments of the same polygon are not always equal, as demonstrated by the polygon on the right hand side of Fig. 3. Here, $\rho\left(u_{w}, u_{x}\right)=\rho\left(D_{r}, B_{r}\right)=r$, whereas $\rho\left(\mathbf{z}_{\mathrm{w}}, \mathbf{z}_{\mathrm{x}}\right)=\rho\left(\mathrm{F}_{\mathrm{l}}, \mathrm{FO}_{\mathrm{l}}\right)=\mathrm{I}$. The circulation direction depends on the position of the reference segment with respect to the other line segments of the same 


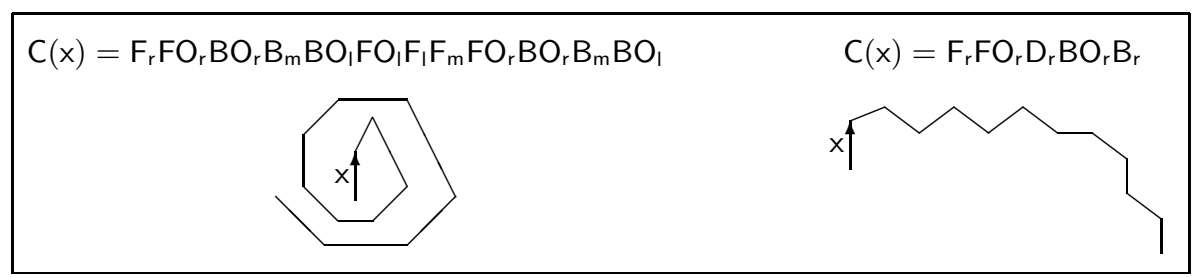

Fig. 6. Polygons without reversals from the point of view of $x$

polygon 2. Changing the circulation direction is what we will focus on in the next section.

\subsection{Reversals}

As soon as a course changes its circulation direction from left to right or right to left, the course includes a reversal, as indicated by its first derivative. For example, $\mathrm{C}(\mathrm{x})=\mathrm{F}_{\mathrm{r}} \mathrm{FO}_{\mathrm{r}} \mathrm{D}_{\mathrm{r}} \mathrm{FO}_{\mathrm{r}} \mathrm{F}_{\mathrm{r}}$ comprises a reversal since the circulation direction is changed after $\mathrm{D}_{\mathrm{r}}$ with the second $\mathrm{FO}_{\mathrm{r}}$ relation.

\section{Definition 3 (Reversal)}

$\mathrm{x}$ is a line segment of a polygon and $\mathrm{C}(\mathrm{x})$ is its course. If $\mathrm{C}(\mathrm{x})$ comprises two sections which circulate in different directions around $\mathrm{x}, \mathrm{C}(\mathrm{x})$ contains a reversal, and it holds $\varrho(\mathrm{C}(\mathrm{x}))$.

Examples of courses without reversals from the viewpoint of line segment $x$ are depicted in Figs. 4 and 6. Examples of courses with exactly one reversal with respect to $x$ are depicted in Fig. 7. As the left hand side of Fig. 5] shows, all reversals of a polygon cannot always be deduced from single courses. It is rather necessary to test all courses of a polygon in order to determine whether there are reversals in the polygon.

An algorithm which determines reversals of a course $C(x)$ derives from

\section{Proposition 2}

$$
\varrho(\mathrm{C}(\mathrm{x})) \Leftrightarrow \exists_{\mathrm{u} \neq \mathrm{x}, \mathrm{v} \neq \mathrm{x}, \mathrm{w} \neq \mathrm{x}}\left(\mathrm{u}<\mathrm{v}<\mathrm{w} \wedge \mathrm{x}_{\mathrm{u}} \neq \mathrm{x}_{\mathrm{v}} \wedge \mathrm{x}_{\mathrm{u}}=\mathrm{x}_{\mathrm{w}} \wedge \eta(\mathrm{C}(\mathrm{x}, \mathrm{u}, \mathrm{w}))<12\right)
$$

Proof: $\mathrm{u}, \mathrm{v}$, and $\mathrm{w}$ are line segments which are unequal to $\mathrm{x}$, and $\mathrm{u}<\mathrm{v}<\mathrm{w}$ ensures an ordering among them, i.e. there is a section, s, of the course $C(x)$ which consists of at least three line segments. As it holds that $x_{u} \neq x_{v}$ there is at least one change in direction in $\mathrm{s}$ while $\mathrm{x}_{\mathrm{u}}=\mathrm{x}_{\mathrm{w}}$ ensures that the first direction in $s$ is again satisfied at the end of $s$. Let $C(x, u, w)$ refer to the section $s$ of $C(x)$ which starts with line segment $u$ and ends with line segment $w$. Then, the course of $s$ must have been turned backwards in the meantime since it holds that the extent of s, i.e. $\eta(\mathrm{C}(\mathrm{x}, \mathrm{u}, \mathrm{w}))$, is less than the extent of the universal scope.

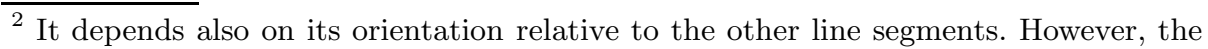
current work solely relies on relative positions and analyses their relationships.
} 


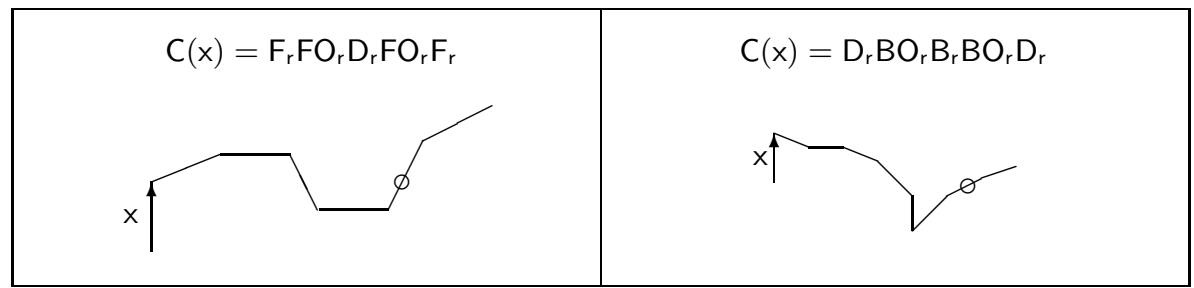

Fig. 7. Polygons with reversals; note that relations are put together when they are equal and adjacent

\subsection{Inflection Segments}

Determining line segments at which reversals occur enables reversals to get distinguished according to $\mathcal{B} \mathcal{A}_{23}$, that is, by describing inflection segments regarding their $\mathcal{B A}_{23}$ relation. Such line segments are defined as follows:

\section{Definition 4 (Inflection segment)}

$\mathrm{x}$ is a line segment of a polygon, $\mathrm{C}(\mathrm{x})$ is its course, and it holds that $\varrho(\mathrm{C}(\mathrm{x}))$. ' $\mathrm{y}$ denotes the predecessor of $\mathrm{y}$ and $\mathrm{y}^{\prime}$ its successor. Then it either holds that $\rho\left(\mathrm{x}^{\prime} \mathrm{y}, \mathrm{x}_{\mathrm{y}}, \mathrm{x}_{\mathrm{y}^{\prime}}\right)=\operatorname{Ir}$ or $\rho\left(\mathrm{x}_{\mathrm{y}} \mathrm{y}, \mathrm{x}_{\mathrm{y}}, \mathrm{x}_{\mathrm{y}^{\prime}}\right)=\mathrm{rl}$. The first line segment after that reversal, namely $\mathrm{y}^{\prime}$, is called the inflection segment.

While $\rho\left(\mathrm{x}_{\mathrm{y}}, \mathrm{x}_{\mathrm{y}}\right)=\mathrm{I}$ and $\rho\left(\mathrm{x}_{\mathrm{y}}, \mathrm{x}_{\mathrm{y}^{\prime}}\right)=\mathrm{r}, \mathrm{y}^{\prime}$ denotes the inflection segment which runs around $x$ the other way round than that line segment which is immediately before $\mathrm{y}^{\prime}$.

Consider, for example, Fig. 7 Inflection segments are marked by circles. Note that these segments are inflection segments from the point of view of reference segment $x$. There are probably other inflection segments when considering other reference segments than $x$. To identify each inflection segment of a polygon it is necessary to analyse all courses, for the same reason as it is necessary to analyse all courses in order to find all reversals.

\section{A Case Study: Differentiating Meanders}

To illustrate the method, we shall compare the similarity of a number of German streams. What we want to know is whether their meanders can be distinguished in determining their reversals, and how the streams relate when ranking them regarding the number of reversals they comprise. For instance, while the Mosel has many twists and turns, the Fulda is less curved but comprises also some windings. Does the concept of reversals as introduced above account for those distinctions?

In order to obtain polygons the rivers have been approximated by the polygonal approximation algorithm of [12]. Using 1:5,000,000 small scale map $\$$, the

\footnotetext{
${ }^{3}$ Note that the images shown are scaled up and down slightly to fit in the layout.
} 


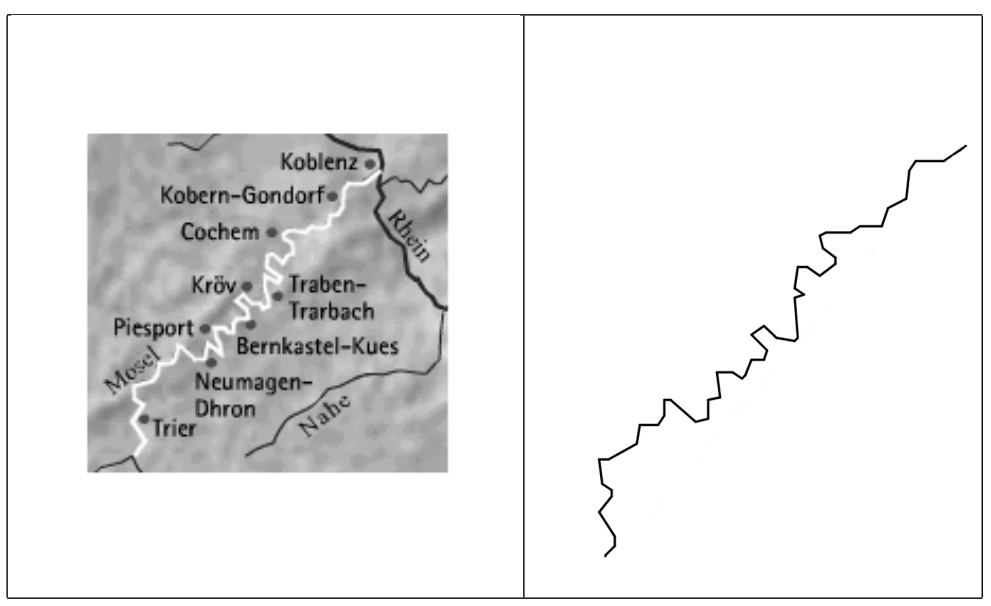

Fig. 8. The river Mosel approximated and simplified by a polygon

Mosel has been approximated by 51 line segments while the Fulda has been approximated by 22 line segments, allowing for the same error in both cases, namely not exceeding a cell in a raster representation. The Fulda comprises 8 reversals (on average, 0.36 per line segment) and the Mosel comprises 94 reversals (on average, 1.84 reversals per line segment). This shows that the method identifies the Mosel as to be five times more curved than the Fulda. But is this still a qualitative difference? It is at least based on qualitative distinctions, namely at the level of $\mathcal{B A}$ relations where we are rather concerned with differences in kind than of measurement. However, counting the number of reversals we obviously turn to some quantitative measurement. On the other hand, the number of reversals involved is in itself without meaning (nor is the normalisation). Instead it is the ordering of those quantities which matters, allowing the streams to get ordered, this ordering being of relative (and hence qualitative) nature.

For the Fulda in Fig. 9 we have printed the inflection segments in pale-grey, in order to visualise how inflection segments appear at those parts of a polygon where indeed large turns are made. Note that such locations can be identified on the basis of qualitative relations alone without requiring precise computations. But instead of precise inflection points of zero curvature we obtain extended portions of the polygon at which the curvature changes.

In Figs. 10 and 11 there are fourteen of the largest streams in Germany and their polygonal approximations. They have been ordered regarding their meanders, i.e. for each stream the number of reversals has been determined and normalised with the number of line segments involved. The polygonal approximations have been printed enlarged in order to allow them to be compared more easily. The first stream with the fewest reversals is the river Havel, that stream with the most reversals is the river Mosel. The shown ordering can in fact be comprehended very well: beginning with the Havel the streams getting more and 


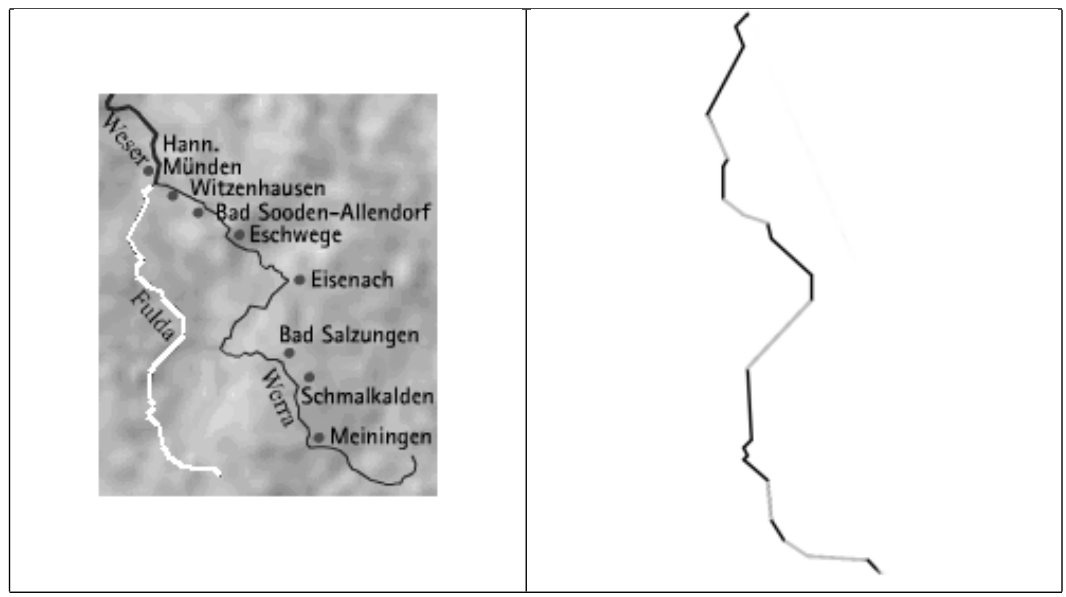

Fig. 9. The river Fulda approximated and simplified by a polygon; inflection segments are printed in pale-grey

more complex. It shows that both little meanders like in the case of the Mosel and larger meanders which extend larger portions of the stream like in the case of the Elbe and the Fulda are equally identified by this method.

\section{Extending the Concept of Neighbourhood Relations}

According to [5] two relations are conceptual neighbours if one of the objects involved can be continuously enlarged, shortened, or moved, so that one relation transforms into the other one without passing any third relation. We shall extend this concept of neighbouring relations by including the circulation direction. That is, while transforming one relation into another one we distinguish whether the line segment which undergoes some deformation circulates around the reference segment clockwise or anticlockwise during this deformation step. This changes nothing about the notion of conceptual neighbourhoods but allows a finer distinction to be made, either during a transformation step or while describing the course of the reference segment.

\section{Definition 5 (Directed neighbourhood relation)}

$\mathrm{x}$ is a line segment of a polygon and $\mathrm{C}(\mathrm{x})$ is its course. Then, two relations, $\mathrm{x}_{\mathrm{y}}$ and $\mathrm{x}_{\mathrm{y}^{\prime}}$, in $\mathrm{C}(\mathrm{x})$ are left-directed neighbours, if they can be directly transformed into one another by continuously deforming (i.e. shortening, lengthening, or moving) $\mathrm{y}$ by circulating left around $\mathrm{x}$ without passing any third relation. Right-directed neighbours are defined accordingly.

This definition adapts to all those qualitative representations which define conceptual neighbourhood graphs, such as [1], 4], and [13. For the time domain, this amounts to distinguish a transformation towards the past and towards the 


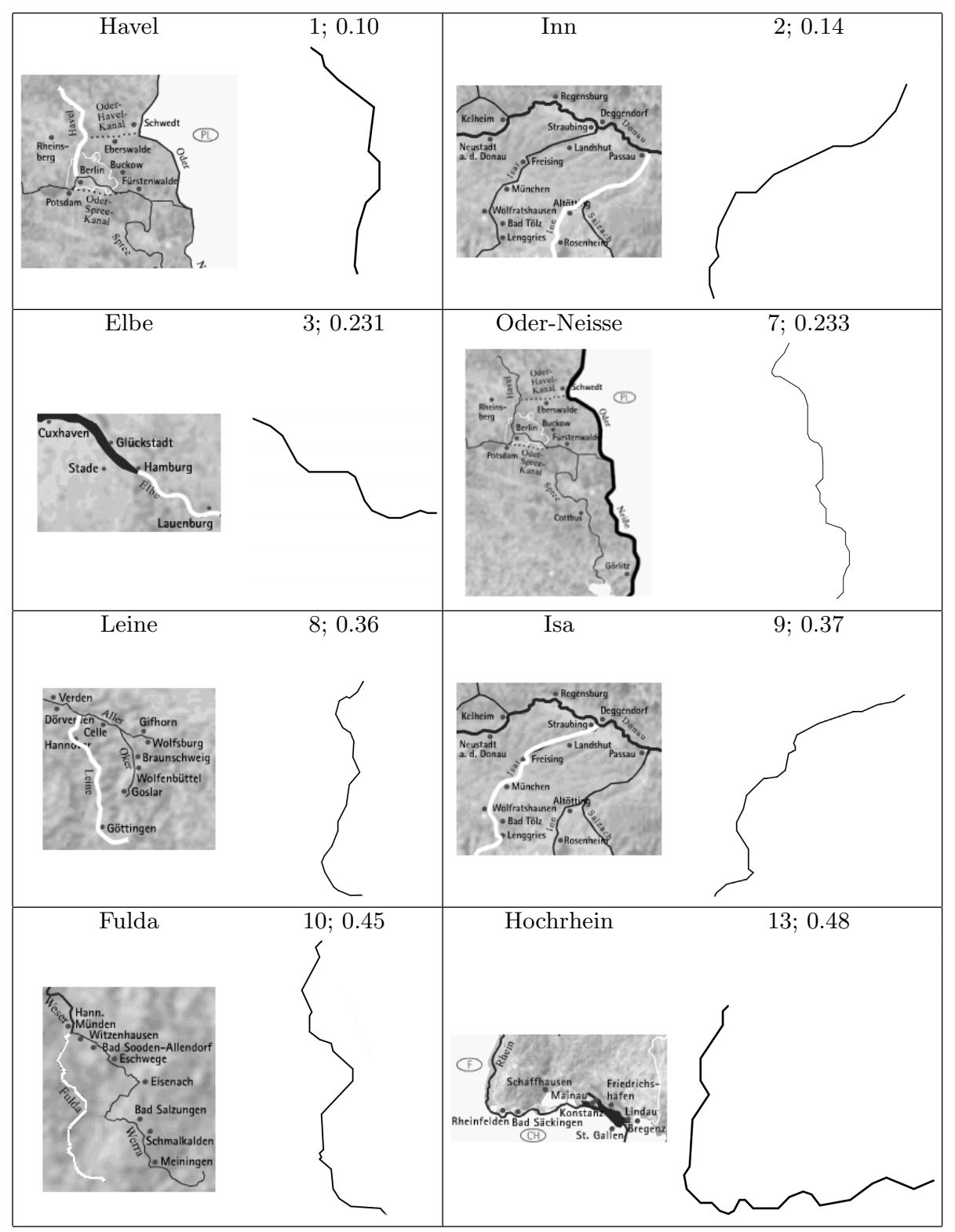

Fig. 10. German streams and their reversals: shown is the absolute number of reversals (first number) and the number of reversals on average per segment (second number)

future; in the domain of regions this allows for the differentiation whether two moving regions merge or separate. Directed neighbourhood relations are uniquely defined for these domains. Informally, this can be read off the neighbourhood 


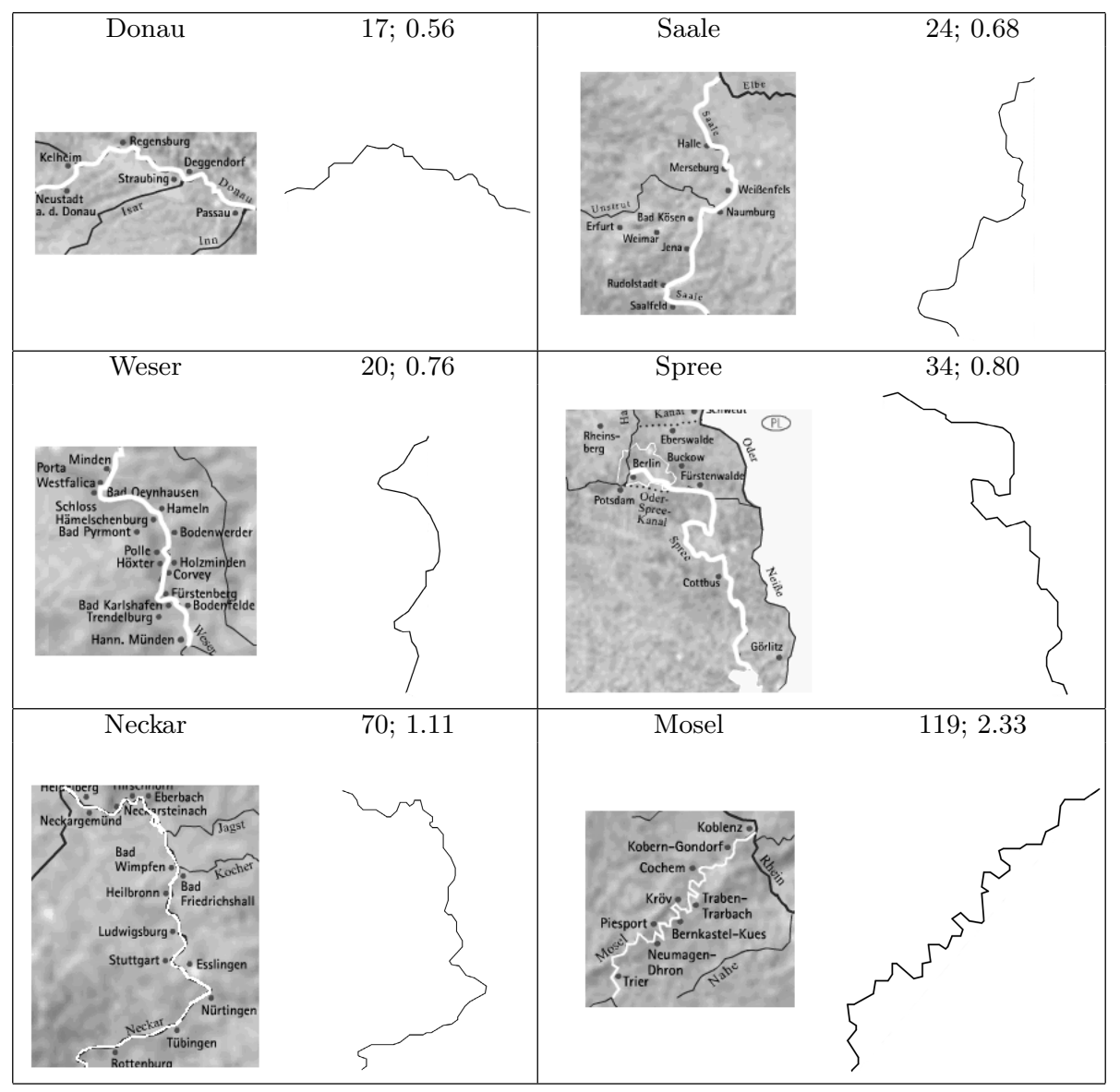

Fig. 11. German streams and their reversals: shown is the absolute number of reversals (first number) and the number of reversals on average per segment (second number)

graphs. In fact, this differentiation is partly implied in the ordering of relations in the time domain, since Allen distinguishes relations such as before and after.

In our case directed neighbourhood relations are necessary in order to distinguish two circulation directions, and as a consequence, in order to be able to recognise reversals as well as inflection segments. But directed neighbourhood relations are yet more fundamental. Qualitative representations frequently define sets of binary [4 or sometimes sets of ternary relations [15] among a number of two or three objects, respectively. But here we are concerned with single objects which are to be characterised qualitatively. The idea behind the qualitative characterisation of single objects relies on the partitioning of objects into parts in order to describe relations among those parts. However, this requires to take into account the ordering of qualitative relations between parts accordingly to how those parts define some object. For instance, concepts such as the circulation direction can 
then be defined, and this makes the distinction between figure and ground possible which requires the orientation of an object's boundary to be determined. In this sense, the concept of directed neighbourhood relations is essential as an additional means for determining specific features among objects or parts.

\section{Discussion}

Having defined generalised directions and reversals, polygons can be classified accordingly. A polygon, for instance, which comprises reversals for neither of its courses would be classified as to be straight. Conversely, the more reversals a polygon has the more curved it is. Describing reversals in combination with generalised directions allow yet finer classifications to be made. Accordingly, sections without reversals can be described by generalised directions. Similarly, inflection segments can be referred to either by $\mathcal{B A}_{23}$ relations or generalised directions.

An advantage of this approach is its ability to cope with indeterminacy: Incompleteness arises if there are gaps in the polygon; nonetheless, all relations between the discernible segments can be used in the described way; this allows to describe the incomplete shape at least partly. Imprecision is dealt with implicitly by the coarseness of $\mathcal{B A}_{23}$ relations. Overall, the strength of these techniques is that the approach is particularly well suited for dealing with coarse shape information. Once precise shape details matter it may be appropriate to apply quantitative methods instead. However, even if information about a stream has been acquired incompletely or imprecisely, it is possible to determine reversals. In order to analyse the robustness of the method, we currently investigate to what extent the chosen precision of the polygonal approximation algorithm affects the number of reversals which are detected.

This generalises to the question of how qualitative features do depend on the granularity level at which polygons approximate linear objects. It is worth investigating to what extent the number of reversals, for example, depends on the chosen granularity level. Whenever there is a part of a course which crosses a singularity this will be the case regardless of whether the course is approximated with many or only with a few line segments. For instance, a course might run first of all left of some reference segment and afterwards in the front of that segment. In this case the course must contain the $\mathrm{FO}_{\text {I }}$ relation somewhere, and this course must contain another $\mathrm{FO}_{\text {I }}$ relation later if it contains a reversal. Dealing with a reversal which is even visible at a coarse approximation level, this same reversal will also be perceivable at each finer granularity level. But things are unfortunately not always that simple: a quite small reversal might be probably smoothed away at coarser granularity levels. However, objects should be approximated at a level of abstraction so that distinctions can be made which are crucial for the application at hand. Otherwise, if it is not possible to find a granularity level on the basis of the given application, objects which are to be compared should at least be approximated at the same granularity level.

The course of a reference segment determines directions at which other line segments are situated relative to the reference segment. However, further 
investigations concern the involvement of line segment orientations and their relations to positional relations, as they have been used in the current work. In particular, it is of interest which properties there are that cannot be defined on positional relations alone. Moreover, the question arises whether the method can be refined so as to make it possible to distinguish small Mosel-like meanders, and large Fulda-like meanders. Eventually, it is of interest to thoroughly analyse how courses of different reference segments of the same polygon are related. For this purpose it might be useful to use the algebraic properties of the $\mathcal{B A}$ relations, as they have been defined in 7 .

\section{Summary}

A qualitative shape representation has been described which allows linear objects to be characterised. This representation is based on arrangements of line segments in the two-dimensional plane, as they especially occur in polygons. The qualitative relations used distinguish both a left-right and a front-back dichotomy, including several intermediate relations such as front-middle and frontoverlap left, for instance. Furthermore, an ordering of the relations is defined in accordance to the ordering of line segments in polygons.

A number of properties of linear objects have been defined on the basis of these relations for the purpose of distinguishing different grades of meanders of linear objects. Applying this method to several German streams shows that they can be ordered regarding their meanders, so that less complex meanders can be distinguished from more complex ones, resulting in an ordering which can be intuitively comprehended. In particular, small meanders and large meanders are both allowed for.

\section{References}

1. J. F. Allen. Maintaining knowledge about temporal intervals. Communications of the ACM, 26(11):832-843, 1983.

2. A. G. Cohn and S. M. Hazarika. Qualitative Spatial Representation and Reasoning: An Overview. Fundamenta Informaticae, 43:2-32, 2001.

3. M. Egenhofer. Query Processsing in Spatial-Query-by-Sketch. Journal of Visual Languages and Computing, 8:403-424, 1997.

4. M. Egenhofer and R. Franzosa. Point-set topological spatial relations. International Journal of Geographical Information Systems, 5(2):161-174, 1991.

5. C. Freksa. Temporal reasoning based on semi-intervals. Artificial Intelligence, 94:199-227, 1992.

6. B. Gottfried. Tripartite Line Tracks, Qualitative Curvature Information. In W. Kuhn, M. Worboys, and S. Timpf, editors, Spatial Information Theory: Foundations of Geographic Information Science, LNCS (2825), pages 101-117. Springer, 2003.

7. B. Gottfried. Reasoning about Intervals in Two Dimensions. In W. Thissen, P. Wieringa, M. Pantic, and M. Ludema, editors, IEEE Int. Conf. on Systems, Man and Cybernetics, pages 5324-5332. IEEE Press, 2004. 
8. B. Gottfried. Singularities in Qualitative Reasoning. In B. Buchberger and J. Campbell, editors, 7th Int. Conf. on Artificial Intelligence and Symbolic Computation, LNAI (3249), pages 276-280, Austria, 2004. Springer.

9. B. Gottfried. Global Feature Schemes for Qualitative Shape Descriptions. In H. W. Guesgen, C. Freksa, and G. Ligozat, editors, IJCAI-05 WS on spatial and temporal reasoning, 2005.

10. E. Jungert. Symbolic spatial reasoning on object shapes for qualitative matching. In A. U. Frank and L. Campari, editors, COSIT 1993, Spatial Information Theory: A Theoretical Basis for GIS., LNCS 716, pages 444-462. Springer, 1993.

11. R. C. Meathrel and A. Galton. A Hierarchy of Boundary-based Shape Descriptors. In B. Nebel, editor, IJCAI 2001, pages 1359-1364, Seattle, Washington, USA, 2001. Morgan Kaufmann.

12. D. A. Mitzias and B. G. Mertzios. Shape Recognition With A Neural Classifier Based On A Fast Polygonal Approximation Technique. Patt. Rec., 27:627-637, 1994.

13. D. A. Randell, Z. Cui, and A. G. Cohn. A spatial logic based on regions and connection. In Proc 3rd Int. Conf. on Knowledge Representation and Reasoning, pages 165-176, San Mateo, 1992. Morgan Kaufman.

14. C. Schlieder. Qualitative Shape Representation. In P. Burrough and A. M. Frank, editors, Geographic objects with indeterminate boundaries, pages 123-140, London, 1996. Taylor \& Francis.

15. K. Zimmermann and C. Freksa. Qualitative Spatial Reasoning Using Orientation, Distance, and Path Knowledge. Applied Intelligence, 6:49-58, 1996. 